SHORT REPORT

\title{
Leucoencephalopathy with neuroaxonal spheroids (LENAS) presenting as the cerebellar subtype of multiple system atrophy
}

M L Moro-de-Casillas, M L Cohen, D E Riley

J Neurol Neurosurg Psychiatry 2004;75:1070-1072. doi: 10.1136/jnnp.2003.028431

Leucoencephalopathy with neuroaxonal spheroids (LENAS) is a rare disease of cerebral and cerebellar white matter. LENAS usually presents as a disorder of cognition and behaviour, or with gait dysfunction and ataxia. This report describes a patient who had a 14 year course of progressive neurological decline consistent with a clinical diagnosis of probable multiple system atrophy, with prominent cerebellar dysfunction and dysautonomia. Formal autonomic laboratory testing was consistent with global autonomic dysfunction of central origin. However, magnetic resonance imaging showed extensive white matter signal abnormalities, in addition to moderate cerebral and cerebellar atrophy. On postmortem microscopic examination, there were numerous axonal spheroids throughout the white matter of both regions. This case of LENAS presented unique clinical characteristics, and typical pathological findings.

L eucoencephalopathy with neuroaxonal spheroids (LENAS) is a progressive disorder of cerebral and - cerebellar white matter with axonal spheroids as its histopathological hallmark. ${ }^{1-4}$ Axonal spheroids are localised swellings of axons composed of structured material, including protein, variable amounts of carbohydrates, lipids, and glycogen. Ultrastructurally, they contain disorganised cellular organelles, neurofilaments, dense granules, microtubules, membrane bound vesicles, and atypical fibrillar material. ${ }^{45}$ Formation of polymorphic axonal spheroids occurs with axonal degeneration, ${ }^{4}$ and may be followed by neuronal loss. Axonal spheroids may occur as a physiological effect of aging, or in association with various pathological processes such as metabolic and degenerative diseases, intoxications, tumours, or trauma. ${ }^{2}$ Clinically, LENAS usually presents with cognitive or behavioural dysfunction, or as a gait disorder and ataxia, with or without corticospinal tract dysfunction. ${ }^{2367}$ Pandysautonomia has not been reported in association with LENAS. Previous reports have included both inherited ${ }^{12}$ and sporadic cases. ${ }^{236}$ We report a case of sporadic LENAS presenting with cerebellar and autonomic dysfunction reminiscent of the cerebellar subtype of multiple system atrophy (MSA-C). ${ }^{8}$

\section{CASE REPORT}

The patient was a 67 year old man with a 10 year history of left hand tremor. In the most recent five years he had developed right hand tremor, gait imbalance, and urinary and faecal incontinence. He reported a variable frequency of falling, up to four times daily, with head concussion on two occasions. He had been impotent for 15 years. Urinary incontinence occurred daily. He had occasional faintness on standing, relieved by sitting down. He had drenching sweats at dinner and at night. His family reported that he had difficulty with memory and concentration. His past medical history included hypertension, benign prostatic hypertrophy, osteoarthritis, and nephrolithiasis; medications included terazosin, paroxetine, and vitamins. There was a history of alcohol abuse 30 years earlier, but no other drug abuse or toxin exposure. There were no risk factors for human immunodeficiency virus or human $\mathrm{T}$ cell leukaemia virus type 1 infection. The family history was notable only for his mother's hand tremor without other neurological deficits. There was no known family history of neurodegenerative disorders, or mental retardation. On neurological examination, he scored 28 out of 30 on a Mini Mental Status examination (MMSE), recalling two of three objects after three minutes. Speech was normal. There was no nystagmus or dysmetria of ocular movement. Pursuit and saccade movements were normal. Cranial nerves were otherwise normal. There was a mild ataxic tremor of the upper limbs, worse on the left, without dysmetria. Tone was normal. Repetitive movements showed slight dysrhythmia. Tendon reflexes were normal. Gait was wide based with moderate difficulty with tandem walking. Postural stability testing was normal.

Magnetic resonance imaging (MRI) showed moderate cerebral and cerebellar hemispheric atrophy, and pronounced centrum semiovale, periventricular, and cerebellar white matter changes (fig lA, B). Autonomic laboratory testing five days after discontinuation of medication disclosed orthostatic hypotension on tilt table testing. His blood pressure decreased by $34 / 14 \mathrm{~mm} \mathrm{Hg}$ at one minute, and by $42 / 34 \mathrm{~mm} \mathrm{Hg}$ at maximum. Cardiac responses to deep breathing and the Valsalva manoeuvre were impaired. The axon reflex sweat test was normal, suggesting intact peripheral autonomic function and implicating a central (preganglionic) localisation.

In the ensuing years, falling and incontinence remained his main problems. He exhibited irritability with outbursts of anger. His wife reported that his short term memory and judgment were impaired, and that his cognitive function had "really hit the skids". He slept excessively. He stopped driving and started choking more. The MMSE was unchanged; he recalled two of three objects at three minutes, and three of three with prompting. The rest of his neurological examination remained stable. He developed dysphagia and aspiration pneumonia. He sustained severe head trauma from a fall down a flight of stairs, and never regained consciousness. He died six days later, at the age of 71, 14 years after the onset of his illness.

Abbreviations: LENAS, eucoencephalopathy with neuroaxonal spheroids; MMSE, Mini Mental Status examination; MRI, magnetic resonance imaging; MSA, multiple system atrophy; MSA-C, type $C$ multiple system atrophy; NAD, neuroaxonal dystrophy 

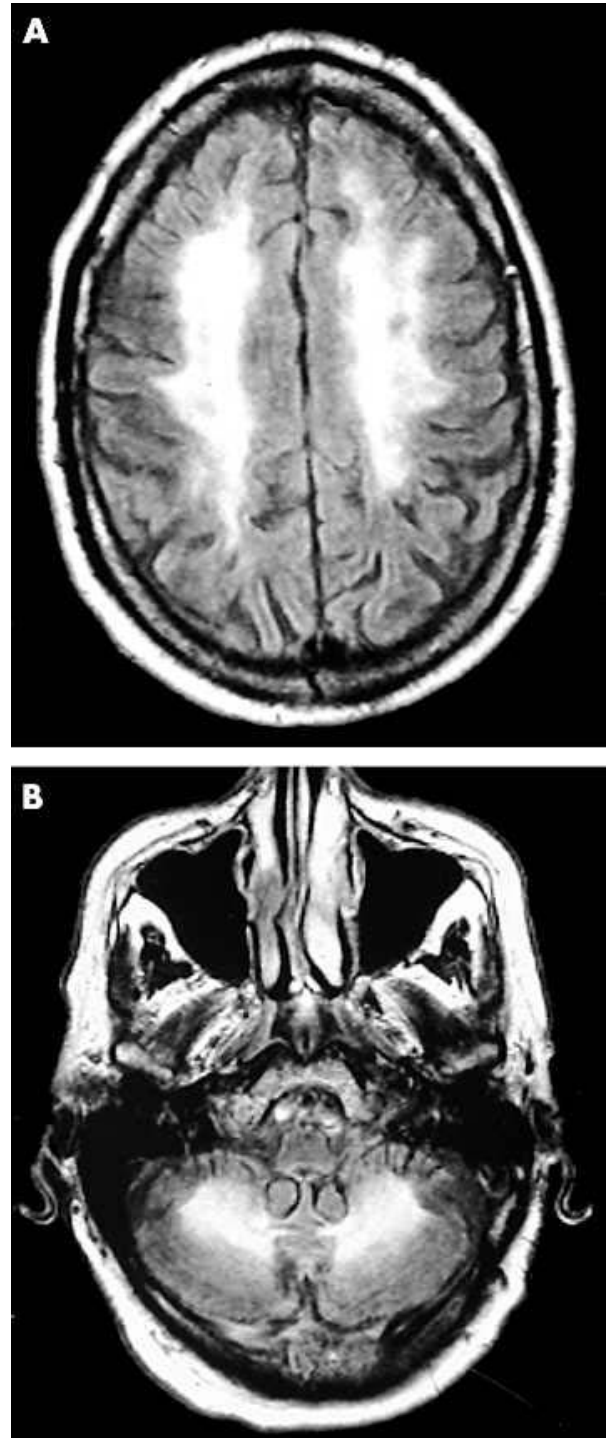

Figure 1 Radiological findings. Fluid attenuated inversion recovery magnetic resonance imaging shows pronounced centrum semiovale and (A) periventricular and (B) cerebellar white matter hyperintensities.

\section{PATHOLOGY}

The necropsy was limited to examination of the brain. The brain weighed $1200 \mathrm{~g}$. The spinal cord was not removed. Gross examination of the brain disclosed $100 \mathrm{ml}$ of subdural blood and a left anterior temporal lobe haematoma. There was no cerebral, cerebellar, or brainstem atrophy. However, the axially sectioned cerebellum was noticeably smaller than that of a 75 year old control cerebellum. Gross inspection of the sectioned cerebral and cerebellar white matter showed patchy discolouration.

Microscopic examination revealed pronounced patchy axonal degeneration in the cerebellar (fig 2A) and cerebral white matter. Perivascular regions appeared relatively spared; however, intimal abnormalities were not present. There were numerous axonal spheroids throughout both regions (fig 2B), as confirmed by neurofilament immunohistochemistry. Cerebellar and cerebral cortices were normal. Axonal spheroids were seen in the substantia nigra, pallidum, and thalamus, but these structures were otherwise normal. Electron microscopy showed relative preservation of myelin surrounding dystrophic axons. There were no arteriolar abnormalities suggestive of cerebral autosomal dominant
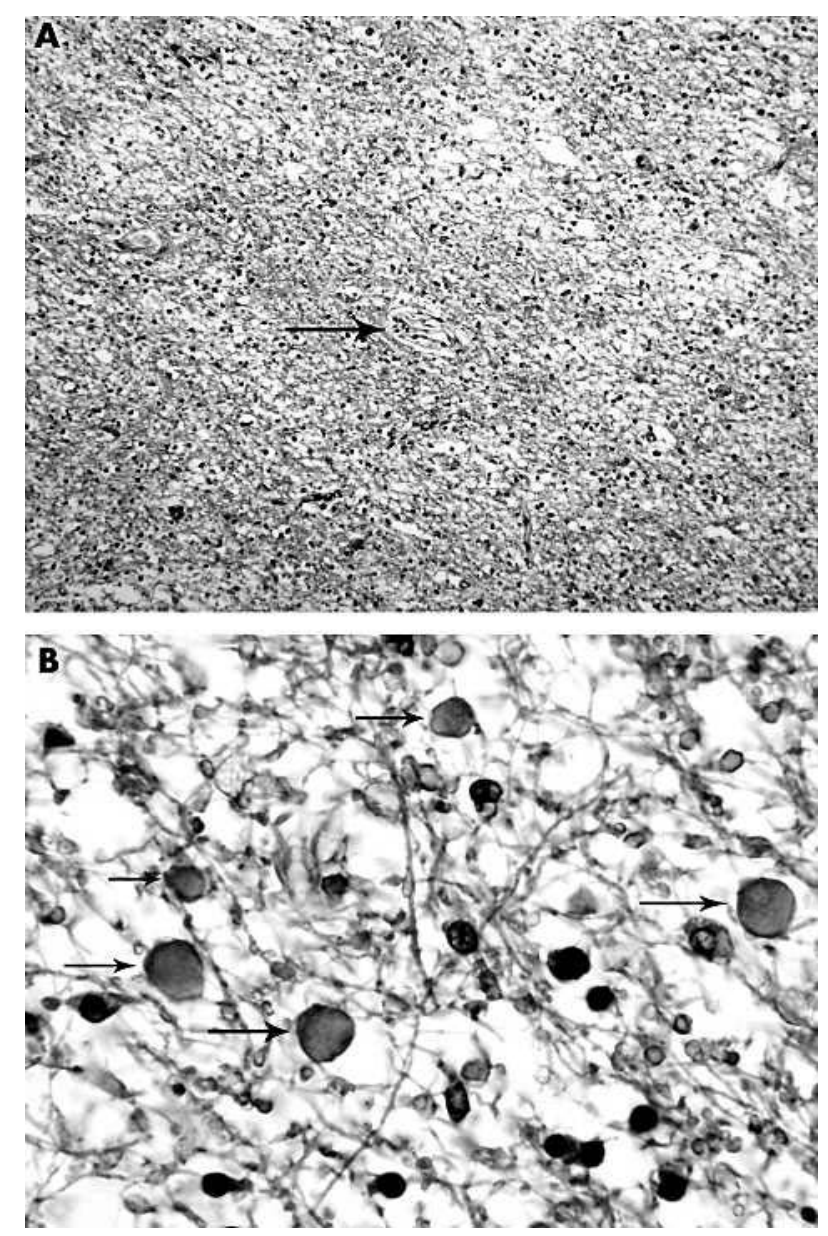

Figure 2 Histopathological findings. (A) At low power, cerebellar white matter shows patchy axonal and myelin loss (haematoxylin and eosin stain; original magnification, $\times 10$ ). Perivascular white matter appears relatively spared (arrow indicates blood vessel). (B) A high power photomicrograph demonstrates numerous axonal spheroids (arrows; haematoxylin and eosin stain; original magnification, $\times 40$ ).

arteriopathy with subcortical infarcts and leucoencephalopathy (CADASIL).

\section{DISCUSSION}

This patient's clinical course was dominated by cerebellar and autonomic dysfunction. These features were consistent with probable MSA-C according to consensus criteria, but his MRI findings (fig 1) excluded this diagnosis. ${ }^{8}$ The protracted course, and the cognitive and behavioural changes reported by his family, were also atypical of MSA. The necropsy findings of widespread patchy white matter disease with numerous axonal spheroids (fig 2) staining positively for neurofilament immunohistochemistry were diagnostic of LENAS.

Axonal spheroids are the pathological hallmark of neuroaxonal dystrophies (NADs). NADs can affect both white and grey matter. In most NADs, such as Seitelberger's disease and Hallervorden-Spatz disease, the primary pathology is in the grey matter. Predominantly white matter abnormalities with axonal spheroids have been found in several diseases including LENAS, polycystic lipomembranous osteodysplasia with sclerosing leucoencephalopathy (Nasu-Hakola disease), and dermatopathy associated neuroaxonal leucodystrophy. ${ }^{35}$ Previous reports of LENAS are few. Axelsson et al described a large Swedish pedigree in which 17 of 71 members were affected in an autosomal dominant fashion, with variable 
penetrance. ${ }^{1}$ The principal clinical features were psychiatric in nature, including depression, anxiety, alcoholism, irritability, and aggressiveness. Seizures, balance and gait disturbances, ataxia, and extrapyramidal symptoms were also part of the disease spectrum. In all four necropsied patients there was pronounced axonal and myelin degeneration, and abundant axonal spheroids in the cerebral white matter. The cerebral cortex was normal. The cerebellum was examined in three of these patients. In two, there was cell loss and axonal swelling in the Purkinje cell layer. The third patient also showed Purkinje cell loss with spheroids in the granule cell layer, white matter of the folia, and dentate nucleus. Goodman and Dickson $^{6}$ reported a patient with sporadic LENAS who had a four year course of dementia, agitation, and aphasia. At necropsy, there was selective degeneration of cerebral white matter characterised by axonal spheroids, many of them vacuolated. The cerebellum was normal.

Van der Knapp and colleagues ${ }^{2}$ reported three cases of LENAS, two from one pedigree and one sporadic patient, whose clinical picture included dementia, behavioural disturbances, spasticity, loss of speech, dysphagia, disequilibrium, and a gait disorder not further described. One patient was said to have ataxia. The pathological findings included white matter degeneration with spheroid formation in the cerebral hemispheres and brainstem; the cerebellum was normal in all patients. Yamashita described a sporadic patient with dementia, and subsequent urinary incontinence and right spastic hemiparesis.. ${ }^{3}$ Pathological changes included atrophy and discolouration of cerebral white matter, axon and myelin loss, and numerous axonal spheroids. The cerebral cortex was normal. In the cerebellum, there were scattered torpedoes in the molecular layer of the cortex, whereas the white matter was normal except for an old infarct. Hancock et al reported a middle aged patient with depressive features, dementia, incontinence, asymmetric corticospinal tract findings, and a broad based gait. ${ }^{7}$ This report presumed that the aetiology was hereditary, although the only affected relatives were two paternal siblings with Alzheimer-type dementia. At necropsy, microscopic white matter examination revealed frequent axonal spheroids, axon loss, and reactive macrophages containing myelin debris. Demyelination was seen within the cerebellum and the brainstem. ${ }^{7}$

Our patient's combination of symptomatic cerebellar and autonomic dysfunction with severe cerebellar white matter degeneration is unique among patients with LENAS. The differential diagnosis of MSA is composed almost entirely of grey matter diseases. ${ }^{9}$ The main exception is multiple sclerosis, which can produce a combination of cerebellar dysfunction and incontinence. To this list, we must now add LENAS, a rare white matter disease that may mimic the clinical presentation of MSA-C.

\section{ACKNOWLEDGEMENTS}

Dr Riley is supported by the Evans Foundation, the estate of Nina Kramer, and the Gift of Nina and Sandy McAfee.

\section{Authors' affiliations}

M L Moro-de-Casillas, D E Riley, Department of Neurology, University Hospitals of Cleveland and Case Western Reserve University School of Medicine, 11100 Euclid Avenue, Cleveland, Ohio 44106, USA

M L Cohen, Department of Pathology, University Hospitals of Cleveland and Case Western Reserve University School of Medicine

Conflicting interests: none declared

Correspondence to: Dr D Riley, Department of Neurology, University Hospitals of Cleveland and Case Western Reserve University School of Medicine, 11100 Euclid Avenue, Cleveland, Ohio 44106, USA; David. Riley@uhhs.com

Received 16 September 2003

In revised form 25 November 2003

Accepted 29 November 2003

\section{REFERENCES}

1 Axelsson R, Royta M, Sourander $P$, et al. Hereditary diffuse leucoencephalopathy with spheroids. Acta Psychiatr Scand Suppl 1984;69(suppl 314):1-65.

2 van der Knaap MS, Naidu S, Kleinschmidt-DeMasters BK, et al. Autosomal dominant diffuse leukoencephalopathy with neuroaxonal spheroids. Neurology 2000;54:463-8.

3 Yamashita M, Yamamoto T. Neuroaxonal leukoencephalopathy with axonal spheroids. Eur Neurol 2002;48:20-5.

4 Seitelberger F. Neuropathological conditions related to neuroaxonal dystrophy. Acta Neuropathol (Berl) 1971;(suppl V):17-29.

5 Lowe J, Lennox G, Leigh PN. Disorders of movement and system degenerations. In: Graham DI, Lantos PL, eds. Greenfield's neuropathology, 6th ed. London: Arnold, 1997:281-366.

6 Goodman LA, Dickson DW. Nonhereditary diffuse leukoencephalopathy with spheroids presenting as early-onset, rapidly progressive dementia [abstract]. J Neuropathol Exp Neurol 1995;54:471.

7 Hancock N, Poon M, Taylor B, et al. Hereditary diffuse leucoencephalopathy with spheroids. J Neurol Neurosurg Psychiatry 2003;74:1345-7.

8 Gilman S, Low PA, Quinn N, et al. Consensus statement on the diagnosis of multiple system atrophy. J Neurol Sci 1999;163:94-8.

9 Gilman S. Multiple system atrophy. In: Jankovic J, Tolosa E, eds. Parkinson's disease and movement disorders. Philadelphia: Lippincott, 2002:170-184. 